\title{
The 4S-AF Scheme (Stroke Risk; Symptoms; Severity of Burden; Substrate): A Novel Approach to In-Depth Characterization (Rather than Classification) of Atrial Fibrillation
}

\author{
Tatjana S. Potpara ${ }^{1,2}$ Gregory Y. H. Lip ${ }^{1,3,4, * \odot}$ Carina Blomstrom-Lundqvist ${ }^{5}$ Giuseppe Boriani ${ }^{6}$ \\ Isabelle C. Van Gelder ${ }^{7}$ Hein Heidbuchel $^{8}$ Gerhard Hindricks $^{9}$ Alan John Camm ${ }^{10}$ \\ ${ }^{1}$ School of Medicine, University of Belgrade, Belgrade, Serbia \\ ${ }^{2}$ Cardiology Clinic, Clinical Centre of Serbia, Belgrade, Serbia \\ ${ }^{3}$ Liverpool Centre for Cardiovascular Science, Liverpool Heart and \\ Chest Hospital, University of Liverpool, Liverpool, United Kingdom \\ ${ }^{4}$ Department of Clinical Medicine, Aalborg University, Aalborg, \\ Denmark \\ ${ }^{5}$ Department of Medical Science, Uppsala University, Uppsala, Sweden \\ ${ }^{6}$ Cardiology Division, Department of Biomedical, Metabolic and \\ Neural Sciences, University of Modena and Reggio Emilia, Modena \\ University Hospital, Modena, Italy \\ 7 University Medical Center Groningen, University of Groningen, \\ Groningen, The Netherlands \\ 8 University Hospital Antwerp, Antwerp University, Antwerp, Belgium \\ ${ }^{9}$ Department of Cardiology and Electrophysiology, University Clinic of \\ Cardiology, Heart Center Leipzig, Leipzig Heart Institute, Leipzig, Germany \\ ${ }^{10}$ St. George's University of London, London, United Kingdom \\ Address for correspondence Tatjana S. Potpara, MD, PhD, Cardiology \\ Clinic, Clinical Centre of Serbia, Visegradska 26, 11000 Belgrade, \\ Serbia \\ (e-mail: tatjana.potpara@med.bg.ac.rs; tanjapotpara@gmail.com).
}

Thromb Haemost 2021;121:270-278.

\author{
Abstract \\ Keywords \\ - atrial fibrillation \\ - classification scheme \\ - temporal-based \\ classification \\ - characterization \\ - stroke risk \\ - symptom severity \\ - burden \\ - substrate
}

Atrial fibrillation (AF) is a complex condition requiring holistic management with multiple treatment decisions about optimal thromboprophylaxis, symptom control (and prevention of AF progression), and identification and management of concomitant cardiovascular risk factors and comorbidity. Sometimes the information needed for treatment decisions is incomplete, as available classifications of AF mostly address a single domain of AF (or patient)-related characteristics. The most widely used classification of AF based on AF episode duration and temporal patterns (that is, the classification to first-diagnosed, paroxysmal, persistent/long-standing persistent, and permanent AF) has contributed to a better understanding of AF prevention and treatment but its limitations and the need for a multidimensional AF classification have been recognized as more complex treatment options became available. We propose a paradigm shift from classification toward a structured characterization of AF, addressing specific domains having treatment and prognostic implications to become a standard in clinical practice, thus aiming to streamline the assessment of AF patients at all health care levels facilitating communication among physicians, treatment

* The editorial process for this paper was fully handled by Prof

Christian Weber, Editor-in-Chief.

received

August 16, 2020

accepted

August 17, 2020

published online

August 24, 2020 (c) 2020. Thieme. All rights reserved. Georg Thieme Verlag KG,

Rüdigerstraße 14,

70469 Stuttgart, Germany
DOI https://doi.org/

10.1055/s-0040-1716408.

ISSN 0340-6245. 
decision-making, and optimal risk evaluation and management of AF patients. Specifically, we propose the 4S-AF structured pathophysiology-based characterization (rather than classification) scheme that includes four AF- and patient-related domainsStroke risk, Symptoms, Severity of AF burden, and Substrate severity-and provide a hypothetical model for the use of $4 \mathrm{~S}-\mathrm{AF}$ characterization scheme to aid treatment decision making concerning the management of patients with $\mathrm{AF}$ in clinical practice.

\section{Introduction}

Ever since the term auricular fibrillation was coined in $1909{ }^{1}$ the understanding of atrial fibrillation (AF) leading to important developments for its management has been constantly evolving, with great improvements having been achieved in the last few decades. ${ }^{2,3}$ The more we learn about AF and its interaction with underlying comorbidity, realizing that it is not just the arrhythmia but also underlying comorbidities that determine outcome, ${ }^{4}$ the greater is our ability to characterize the clinical profile of each patient to provide information relevant for optimal management decisions.

Currently, international AF guidelines and consensus documents list multiple classifications of AF (-Table 1), ${ }^{5-9}$ each classification addressing a single domain of AF (or patient)-related characteristics. Although these classifications have been gradually evolving toward better precision and clinical utility, ${ }^{10}$ they separately address specific features relevant for the arrhythmia, the patient or the clinical setting in which AF occurs. Lack of integration becomes burdensome and sometimes the information needed for treatment decisions is incomplete.

The most widely used classification of AF based on AF episode duration and temporal patterns (that is, the 3-P classification to first-diagnosed, paroxysmal, persistent/ long-standing persistent, and permanent AF, depending on the duration of the arrhythmia and its mode of termination) was proposed by Gallagher and Camm in $1997^{11,12}$ and formally adopted by the European Society of Cardiology Working Group on Cardiac Arrhythmias in $1998 .{ }^{13}$ Before

Table 1 Classifications of AF in current international AF guidelines

\begin{tabular}{|c|c|c|c|c|}
\hline & \multicolumn{4}{|c|}{ Major international AF guidelines } \\
\hline AF classification & $2016 \mathrm{ESC}^{6}$ & $2019{\mathrm{AHA} / \mathrm{ACC} / \mathrm{HRS}^{7}}^{7}$ & $2018(2014) \mathrm{CCS}^{8}$ & 2018 NHFA/CSANZ $^{9}$ \\
\hline \multicolumn{5}{|l|}{ Temporal pattern } \\
\hline & $\begin{array}{l}\text { - First diagnosed } \\
\text { - Paroxysmal } \\
\text { - Persistent } \\
\text { - Long-standing persistent } \\
\text { - Permanent }\end{array}$ & $\begin{array}{l}\text { - First diagnosed } \\
\text { - Paroxysmal } \\
\text { - Persistent } \\
\text { - Long-standing } \\
\text { - Persistent } \\
\text { - Permanent }\end{array}$ & $\begin{array}{l}\text { - First diagnosed } \\
\text { - Paroxysmal } \\
\text { - Persistent } \\
\text { - Permanent }\end{array}$ & $\begin{array}{l}\text { - First diagnosed } \\
\text { - Paroxysmal } \\
\text { - Persistent } \\
\text { - Long-standing } \\
\text { - Persistent } \\
\text { - Permanent }\end{array}$ \\
\hline Symptom severity & $\begin{array}{l}\text { EHRA Symptom severity score: } \\
\text { - I No symptoms } \\
\text { - Ila Mild } \\
\text { - IIb Moderate } \\
\text { - III Severe } \\
\text { - IV Disabling }\end{array}$ & $\begin{array}{l}\text { - Asymptomatic } \\
\text { (silent) } \\
\text { - Symptomatic }\end{array}$ & $\begin{array}{l}\text { SAF score: } \\
\text { - } 0 \text { (asymptomatic) } \\
\text { - } 1 \text { (minimal impact on QoL) } \\
\text { - } 2 \text { (minor impact on QoL) } \\
\text { - } 3 \text { (moderate impact on QoL) } \\
\text { - } 4 \text { (severe impact on QoL) }\end{array}$ & $\begin{array}{l}\text { - Asymptomatic } \\
\text { (silent) } \\
\text { - Symptomatic }\end{array}$ \\
\hline \multicolumn{5}{|l|}{$\begin{array}{l}\text { Underlying } \\
\text { comorbidity }\end{array}$} \\
\hline - Valvular AF & $\begin{array}{l}\text { Replaced with "AF in } \\
\text { patients with MS or } \\
\text { prosthetic heart valves" }\end{array}$ & Yes & Yes & Yes \\
\hline $\begin{array}{l}\text { Clinical type reflecting } \\
\text { different causes of AF }\end{array}$ & $\begin{array}{l}\text { - Secondary to structural } \\
\text { heart disease } \\
\text { - Focal } \\
\text { - Polygenic / Monogenic } \\
\text { - Postoperative } \\
\text { - In athletes } \\
\text { - }\end{array}$ & No specific list & No specific list & No specific list \\
\hline $\begin{array}{l}\text { Surface ECG } \\
\text { appearance } \\
\text { Coarse / Fine }\end{array}$ & No & No & No & No \\
\hline $\begin{array}{l}\text { Mode of onset } \\
\text { Vagal / Adrenergic }\end{array}$ & No & No & No & No \\
\hline
\end{tabular}

Abbreviations: ACC, American College of Cardiology; AF, atrial fibrillation; AHA, American Heart Association; CCS, Canadian Cardiovascular Society; ECG, electrocardiogram; EHRA, European Heart Rhythm Association; ESC, European Society of Cardiology; HRS, Heart Rhythm Society; MS, mitral stenosis; NHFA/CSANZ, National Heart Foundation of Australia / Cardiac Society of Australia and New Zealand; QoL, quality of life; SAF, symptom severity in AF. 
this, Lévy et al had redefined paroxysmal AF as lasting no more than 7 days and terminating spontaneously. ${ }^{14}$ The temporal pattern-based classification of AF has contributed to a better understanding of AF prevention and treatment but its limitations and the need for a multidimensional AF classification are being increasingly recognized. ${ }^{15}$

Importantly, the pattern-based classification of AF provided standardization of AF-related nomenclature and was easily adopted owing to its simplicity. However, despite generally correlating with the extent of the atrial substrate, remodeling, and AF-related outcomes, the pattern-based classification of AF lacks precision in differentiating among specific features relevant for optimal treatment decisions with regards to stroke prevention and rhythm control strategies such as catheter ablation. ${ }^{16}$ Indeed, formal recommendations for the management of AF are not based on the pattern of AF, except for the decision to restore sinus rhythm, but the terms paroxysmal and persistent AF include a large conglomerate of patients with wide variations in AF patterns, substrates, and other characteristics and, consequently, different needs in management. Owing to the availability of continuous rhythm monitoring, we learned only recently that patients classified to the same clinical AF category may be inherently heterogeneous in terms of temporal AF persistence and AF burden. ${ }^{17,18}$ The differentiation between paroxysmal and persistent AF is often very cumbersome, both patterns may be observed in the same patient during follow-up, and the heterogeneity in recurrences and progression of AF poses a challenge to a rhythm-based classification. Also, it describes only the arrhythmia, whereas other relevant features such as cardiovascular risk factors and underlying comorbidities or the extent of atrial substrate changes are not included. ${ }^{6}$

In 2010, Lubitz et al proposed a more extensive classification of the arrhythmia, ${ }^{19}$ and the multidimensional form of classification of AF was presented in more details by Camm et al in $2012 .{ }^{15}$ Given the multiplicity of factors relevant for optimal management of $\mathrm{AF}$ in clinical practice, including advances in monitoring of AF and risk assessment tools, and evolving treatment options apart from the complexity of AF itself, a simple but comprehensive characterization of AF is urgently needed.

From this viewpoint, we propose a paradigm shift from classification toward a structured characterization of $\mathrm{AF}$ addressing specific domains that have treatment and prognostic implications to become a standard in clinical practice, thus streamlining the evaluation of AF patients at all health care level, with the goal to facilitate communication among physicians, treatment decision-making, and optimal management of AF patients.

Specifically, we propose the 4S-AF structured pathophysiology-based characterization (rather than classification) scheme that includes four AF- and patient-related domains (Stroke risk; Symptoms; Severity of AF burden; Substrate severity)( - Fig. 1).

\section{The Need for a Structured AF Characterization}

Being a multifaceted, complex, and very heterogeneous disease, AF requires structured patient management and multiple treatment decisions addressing different treatment domains such as stroke prevention, symptom improvement, and management of concomitant conditions and risk factors. ${ }^{6,20}$ Importantly, these decisions should be regularly reevaluated, owing to dynamic changes in the patients' individual risk profile. ${ }^{21,22}$

Sometimes the complexity of patient- and/or AF-related features requires multidisciplinary engagement to facilitate treatment decisions relating to thromboembolic protection,

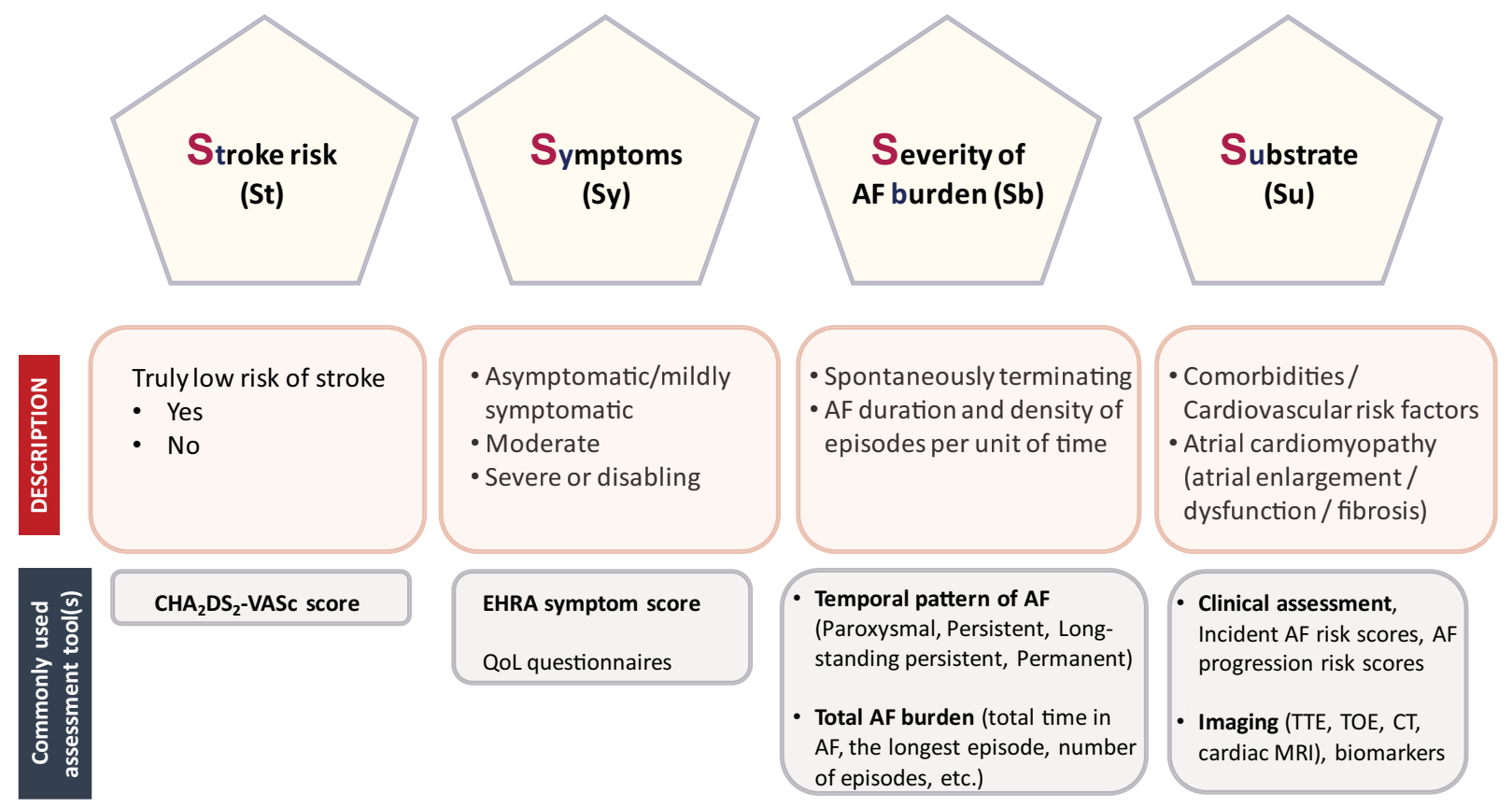

Fig. 1 The 4S-AF scheme for characterization of patients with atrial fibrillation. AF, atrial fibrillation; CT, computed tomography; EHRA, European Heart Rhythm Association; MRI, magnetic resonance imaging; QoL, quality of life; TOE, transesophageal echocardiogram; TTE, transthoracic echocardiogram. 
cardioversion, antiarrhythmic drug therapy, left atrial catheter ablation, or rate control. In daily clinical work, AF-related communication among practitioners including expert consultants needs to be rapid but comprehensive, efficiently providing all the relevant information to facilitate treatment decisions. ${ }^{10}$

Modern medicine is characterized by rapidly evolving means of communication of health-related information among physicians (and patients), including computer- or mobile application-based decision support tools for clinicians and/or patients. Recently, several such tools have been developed specifically for AF, and preliminary data suggest a potential for improving AF management and patient outcomes using these tools. ${ }^{5,23,24}$ Importantly, their output strongly depends on comprehensiveness (and accuracy) of AF-related and other health information entered for a given patient.

Moreover, the use of electronic medical and health records is increasing worldwide. These systems provide an opportunity for the rapid creation of large data sets that can be used for research purposes. ${ }^{25}$ The use of a uniformly structured characterization of AF patients across various data sets would further improve the compatibility of data from various sources.

In all these circumstances, including routine clinical practice, the use of a more structured characterization of $\mathrm{AF}$ patients, with well-defined descriptors would facilitate not only the communication among involved practitioners but also treatment decisions and overall management of $\mathrm{AF}$ patients, ${ }^{20}$ ultimately improving outcome for the individual patients and health care costs.

Indeed, using such a structured AF patient characterization scheme would help physicians achieve a good balance of simplicity, practicality, and information-based treatment decision-making.

\section{The 4S-AF Scheme for Characterization of Patients with Clinically Diagnosed AF}

In addition to identification and management of cardiovascular risk factors and underlying comorbidity, the two key AF-specific treatment decisions are (1) the need for thromboprophylaxis to prevent stroke or systemic embolic events, most commonly the choice of oral anticoagulant therapy (OAC), and (2) appropriate choice of rate and/or rhythm control to improve symptoms and prevent complications of AF such as heart failure. ${ }^{20}$ In addition, the rhythm control treatment strategy often involves choosing between longterm antiarrhythmic drug medication or AF ablation therapy (i.e., surgical or catheter ablation). ${ }^{6}$

Whereas patient's age (and other demographic features), cardiovascular risk factors, and comorbid diseases should be routinely noted in the patient's medical record, a more detailed characterization of AF-specific features and patient-related characteristics combined into a structured system for a comprehensive description of AF would facilitate the choice of optimal treatment (including interventional procedures), not only influencing the success of interventional procedures but also improving patient outcome.

We, therefore, propose a structured characterization of AF patients in clinical practice using the $4 \mathrm{~S}-\mathrm{AF}$ scheme that addresses Stroke risk, Symptom severity, Severity of AF burden, and Substrate for AF to be used in clinical practice (-Fig. 1). This approach is based on the principles generally similar to the most widely used tumor clinical staging system (i.e., the TNM tumor classification) but unlike tumors, AF would be characterized rather than staged.

The 4S-AF scheme would provide essential information needed for decision-making on the use of OAC, choice of rate or rhythm control and between AF ablation or antiarrhythmic medication, and treatment of underlying cardiovascular comorbidities and risk factors. At this point, we do not propose this system as a definitive treatment-decision tool, since data on its association with treatment outcomes are currently lacking, but rather as a structured descriptive aid in the decision-making process. Nevertheless, we certainly do not exclude that with the acquisition of data in the future and evaluation of the proposed characterization scheme, this might become the case.

\section{The 4S-AF Scheme Domains}

The Stroke risk (St) domain characterization is currently based on the routinely used and guideline-recommended clinical risk factor-based $\mathrm{CHA}_{2} \mathrm{DS}_{2}$-VASc score for stroke risk assessment, and the indication for OAC use is established as per guideline recommendations from the European Society of Cardiology and other international bodies (-Fig. 1). ${ }^{6}$

Multiple blood biomarkers (e.g., B-type natriuretic peptide, cardiac troponin, biomarkers of renal function, etc.) and indices of atrial structural and functional remodeling obtained by various imaging tools have been shown to correlate with individual AF-related thromboembolic risk, and several biomarker-based stroke risk scores ${ }^{26}$ have been validated, showing modest but statistically significant improvement in stroke risk prediction when biomarkers are added to clinical risk factors. ${ }^{27}$

Recent evidence suggest that the burden of AF may be associated with thromboembolic risk and all-cause mortality. ${ }^{28}$ Whereas the landmark trials of nonvitamin K antagonist oral anticoagulants versus warfarin for stroke prevention in AF consistently showed that the residual thromboembolic risk among anticoagulated patients was significantly lower in those with paroxysmal as compared with persistent AF even after adjustment for baseline characteristics, ${ }^{29}$ data from earlier trials that included nonanticoagulated controls $^{30}$ and contemporary AF registries or population-based studies $^{31,32}$ are conflicting.

While the clinically assessed burden of AF using only intermittent electrocardiographic (ECG) monitoring might not significantly impact stroke risk (that is, clinically evident AF has already crossed the threshold for elevated stroke risk), an increasing body of evidence derived from cohorts of patients implanted with pacemakers or defibrillators capable of continuous heart rhythm monitoring or a high-risk cohort of patients with insertable cardiac monitors or wearable monitoring devices suggests that even the burden of subclinical AF could impact the risk of stroke. ${ }^{33}$ However, it remains to be clarified whether stroke risk is a continuum or there is a specific threshold of AF burden at which the risk significantly increases. 
Owing to the rapidly advancing technologies for computer-based decision support tools and machine learning application in medicine, the description of stroke risk in $\mathrm{AF}$ patients may evolve beyond clinical risk factor-based approach, but the decision to use OAC will always be a binary yes/no entity. ${ }^{34}$

The Symptom severity (Sy) domain addresses the patientcentered, symptom-directed focus of AF management. This component focuses on the severity of symptoms, currently using the European Heart Rhythm Association (EHRA) symptom score, and is important for treatment decisions. ${ }^{6,20}$ of note, the EHRA symptom severity score is prognostically relevant for adverse cardiovascular events. ${ }^{35}$

However, the EHRA symptom score is physician-assessed, reflecting how physicians weigh the symptoms of their AF patients rather than the patients' perception that may differ substantially. ${ }^{36}$ In addition, the EHRA score cannot precisely differentiate between AF-related and concomitant comorbidity-related symptoms. Indeed, in symptomatic patients with underlying comorbidities, optimal management of concomitant diseases is necessary before proceeding with a symptomguided treatment decision for left atrial ablation, and the descriptor(s) of the symptom severity domain may change in the future to include the assessment of quality of life, patient-perceived burden of treatment, ${ }^{37}$ and other features.

The Severity of AF burden (Sb) domain characterizes the proportion of time spent in AF and density of AF episodes in time (if the arrhythmia is not permanent), including also the mode of termination (spontaneously terminating or not) as a potential indicator of the propensity toward the development of chronic arrhythmia.

As mentioned, the clinical adjudication of AF burden using the classification to paroxysmal, persistent, or permanent $\mathrm{AF}$ may be imprecise in distinguishing between paroxysmal and persistent AF (and, occasionally, even permanent AF), which may influence the selection of patients suitable for specific $\mathrm{AF}$ ablation procedures. As exemplified in - Fig. 2 , the outcome of antiarrhythmic drug therapy varies widely even within similar patient groups with the same type of AF in randomized clinical trials comparing AF ablation versus antiarrhythmic medication (control arm), which underlines the importance of finding better tools for characterization of AF features in clinical decision making. The 4S-AF scheme proposes an empirical, clinical assessment-based semiquantification of AF burden that reflects elements relevant for treatment decision with regards to rhythm control, antiarrhythmic drug therapy, and catheter ablation (-Figs. 1 and $\mathbf{3}$ ).

In routine practice, clinicians may roughly assess the duration of symptomatic AF episodes and history of AF via detailed history taking and intermittent ECG monitoring. Thus, AF episodes lasting for hours to days can be arbitrarily labeled as short, those lasting weeks to months would be intermediate, and long AF episodes would be those lasting 12 or more months. The density of AF episodes can be expressed as the annual number of episodes, for example, up to 1 to 3 per year (infrequent), $>3$ per year (frequent), or occurring

\section{One-year freedom from AF}

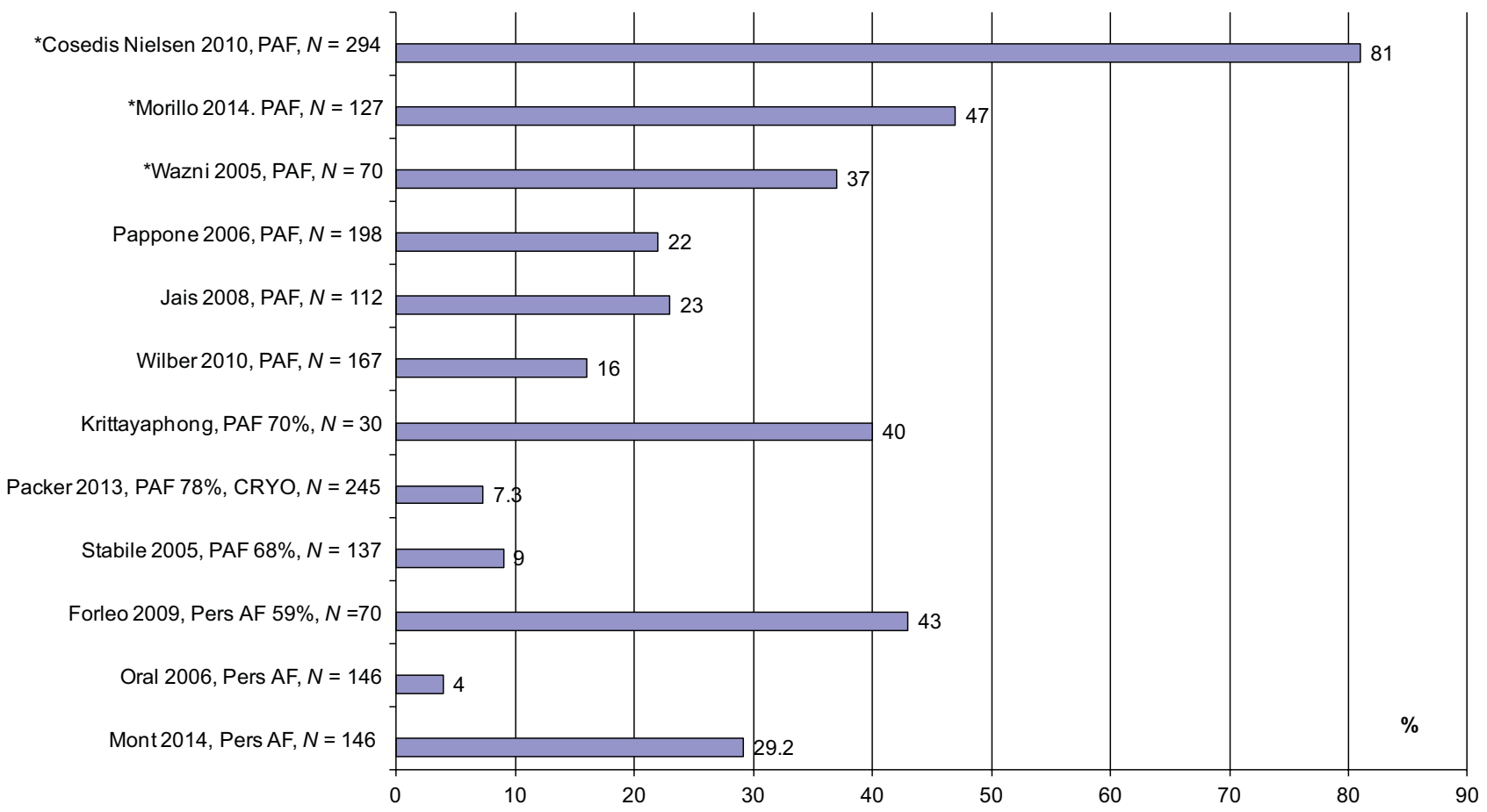

Fig. 2 Atrial fibrillation recurrence rates in the control arms (antiarrhythmic drugs) in various randomized trials comparing antiarrhythmic drugs and atrial fibrillation ablation. ${ }^{50-60}$, *AF ablation as first-line therapy; N, number of patients in the trial; PAF, paroxysmal atrial fibrillation; Pers, persistent atrial fibrillation. Note the disparate outcomes in the control arms in the various trials despite similar AF types in randomized trials comparing antiarrhythmic drugs and AF ablation. Most studies included mainly paroxysmal AF patients and evaluated AF ablation as secondary treatment. Percentages in text denote the dominating AF type. The bars denote the \% of freedom from AF at 12 months. 

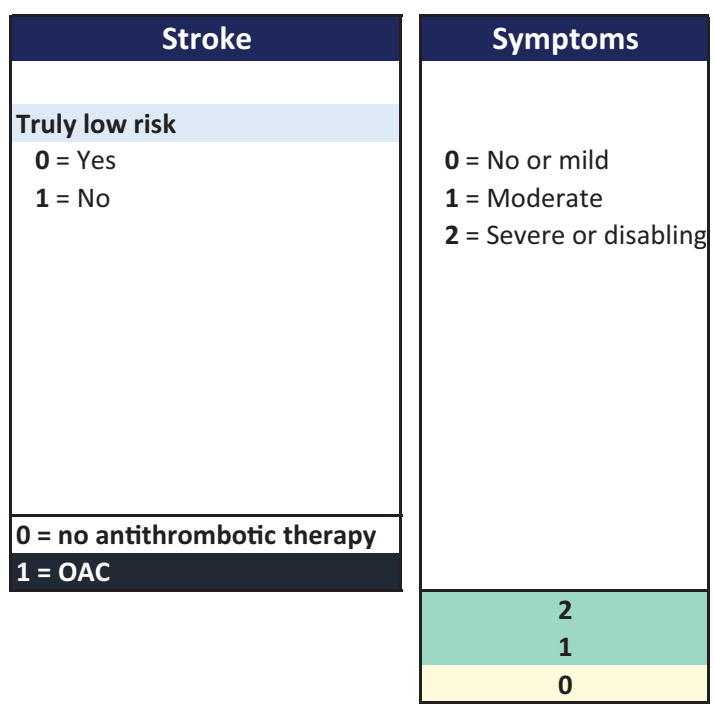

Current commonly used tools for assessment of the 4S-AF domains

EHRA symptom score Temporal pattern of AF

CHA2DS2-VASc score
Truly low risk: 0 males, 1 females

OAC: $>=1$ males , >=2 females

EHRA $1-2 a=0$

EHRA $2 b=1$

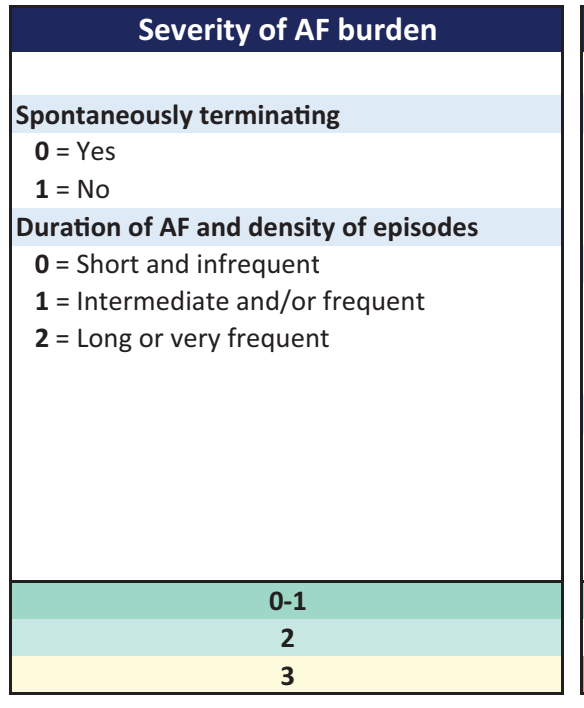

\begin{tabular}{|c|}
\hline Substrate \\
\hline$(+1$ if $>75$ years old) \\
\hline Comorbidity/CV risk factors \\
\hline $\mathbf{0}=\mathrm{No}$ \\
\hline $1=$ Single \\
\hline 2 = Multiple (2 or more) \\
\hline LA enlargement/dysfunction \\
\hline $0=\mathrm{No}$ \\
\hline 1 = Mild-moderate \\
\hline $\mathbf{2}=$ Severe \\
\hline LA fibrosis \\
\hline $0=\mathrm{No}$ \\
\hline $1=$ Mild \\
\hline 2 = Moderate-severe \\
\hline $0-2$ \\
\hline 3-4 \\
\hline 5 or more \\
\hline
\end{tabular}

EHRA 3-4 =2

\section{Paroxysmal or first onset $=0$}

Persistent $=1$

Long-standing persistent $=2$

Permanent $=2$

\section{Various}

Clinical assessment

Transthoracic echocardiography AF progression risk scores

Advanced imaging Biomarkers

All green => Rhythm control
1 Yellow, 2 Green => Rhythm control can be attempted
Red => Consider rate control
Consultation or rate control

Fig. 3 A hypothetical treatment decision supporting algorithm using the 4S-AF scheme for characterization of patients with atrial fibrillation in clinical practice. AF, atrial fibrillation; CV, cardiovascular; LA, left atrium; OAC, oral anticoagulant therapy.

daily to monthly (very frequent). Given that this is only an arbitrary, not validated stratification, the widely adopted and guideline-recommended temporal pattern-based classification of AF can be used initially.

The assessment of AF burden is evolving in parallel with rapidly advancing wearable and insertable technologies for prolonged monitoring of $\mathrm{AF}$ that are becoming increasingly affordable and convenient for long-term use. ${ }^{18}$ Hence, the description and grading of AF burden will likely change in the near future as our knowledge on the association of outcomes with AF burden increases (for example, a good correlation of AF burden with quality of life has been recently reported). ${ }^{38}$

The Substrate for AF (Su) domain pertains to the complexity of AF pathophysiology, including simple clinical characteristics such as patient age, cardiovascular risk factors (e.g., obesity), and underlying comorbidities, as well as the presence and extent of left atrial enlargement, impaired atrial function, and fibrosis of the atrial myocardium, all of which have been shown to play a role in the development and progression of $\mathrm{AF}^{39}$

Diagnostic assessment for the presence of cardiovascular risk factors and underlying comorbidities is not only a routine part of comprehensive clinical evaluation of any patient suspected of having a heart condition but is also highly relevant for treatment decisions with regards to thromboprophylaxis and likelihood of successful rhythm control. Of note, the cardiovascular risk factor burden closely correlates with the lifetime risk of AF development, ${ }^{40}$ whereas optimal management of modifiable cardiovascular risk factors and comorbidities has been associated with a reduction in AF burden. ${ }^{41}$ Indeed, the structured characterization of AF using the $4 \mathrm{~S}-\mathrm{AF}$ scheme would prompt practicing physicians to identify and manage these risk factors, whereas the acknowledgment of multimorbidity that is included in the $4 \mathrm{~S}$-AF would influence the arrhythmia-related treatment decisions.

Atrial structural and functional remodeling predisposing to or resulting from $\mathrm{AF}$ is an important indicator of substrate complexity and correlates well with the outcome of AF-directed treatment interventions. ${ }^{42}$ Of note, left atrial size and function generally correlate well with cardiovascular outcomes including all-cause mortality. ${ }^{43,44}$ Transthoracic echocardiography is widely available in routine clinical practice and provides basic information on the atrial size and function, whereas more sophisticated assessment including advanced transthoracic echocardiography, transesophageal echocardiography, cardiac computed tomography, or nuclear magnetic resonance imaging provides additional indices of atrial dysfunction and structural alterations including fibrosis ${ }^{42}$ and epicardial fat that have both treatment and prognostic implications and may inform expert decision-making (e.g., choosing the appropriate ablation strategy). ${ }^{45}$ The rapidly advancing technologies such as machine learning may also enhance the 
characterization of correlates for atrial structure in the risk assessment of AF recurrence. ${ }^{46}$

With increasing evidence about the clinical application of the recently proposed concept of atrial cardiomyopathy, ${ }^{47}$ the assessment, classification, and staging of the atrial disease may become the cornerstone of the Su domain in the 4S-AF scheme.

\section{The Use of the 4S-AF Scheme for Characterizing AF Patients-Hypothetical Examples in Clinical Practice}

The 4S-AF characterization of AF patients using the descriptors obtained by routine diagnostic assessment ( - Figs. 1 and 3) would provide the basis for treatment decision-making supporting optimal management of AF by primary care physicians, internal medicine specialists, or general cardiologists, also facilitating optimal referral for expert consultation where needed ( - Fig. 3), whereas further refinement of AF characterization using advanced diagnostic imaging tools would facilitate expert decision-making.

Importantly, the $4 \mathrm{~S}-\mathrm{AF}$ characterization of AF patients must be accompanied by information on the patient age and comorbidities and personal preferences.

Example 1: The 4S-AF characterization is $S t=0, S y=2$, $S b=3, S u=0$, and the medical report describes a female patient aged 59 years, with no cardiovascular or other comorbidity besides AF.

It is immediately obvious that this patient does not need long-term OAC (low stroke risk) but is eligible for rhythm control including consideration for AF ablation as a first-line treatment owing to severe symptoms, high AF burden, and low substrate complexity. ${ }^{6}$

Warning: this patient would still need OAC before and after AF ablation, as recommended, and her $4 \mathrm{~S}-\mathrm{AF}$ status should be reassessed regularly in order not to miss dynamic changes in her risk profile over time. ${ }^{6}$

Example 2: The 4S-AF characterization is $S t=1, S y=0$, $S b=3, S u=4$. As per the medical report, it is a male patient, 79 years old, with prior stroke, myocardial infarction, diabetes mellitus, and hypertension, whereas transthoracic echocardiogram showed preserved left ventricular function and a left atrial anteroposterior diameter of $48 \mathrm{~mm}$ with mild mitral regurgitation.

We can easily appreciate that this patient has a high risk of stroke, mild symptoms, high AF burden (i.e., long-standing persistent or permanent AF; see - Fig. 3), and significant substrate for AF. Hence, the patient needs lifelong OAC, and rate control may be the ultimate solution, which should be discussed with the patient weighing the risk factors for AF recurrence. Again, the $4 \mathrm{~S}$ status of this patient should be reviewed regularly. Also, the patient should be routinely monitored for adherence to treatment, the occurrence of AF-related complications (e.g., stroke, bleeding, heart failure), and comorbidities. ${ }^{6}$

Example 3: A 63-year-old female patient is referred for expert consultation for further AF management after electrical cardioversion failure in the local hospital. Her $4 \mathrm{~S}-\mathrm{AF}$ characterization is $S t=1, S y=2, S b=3, S u=5$. She is obese, has hypertension, bilateral aortic-femoral graft, and chronic obstructive pulmonary disease, and severely increased left atrium volume $\left(42 \mathrm{~mL} / \mathrm{m}^{2}\right)$ on transthoracic echocardiographic examination. The 12-lead ECG shows AF with a ventricular rate of 125 beats per minute.

The patient is highly symptomatic but $S u=5$ suggests advanced substrate for AF. Indeed, her symptoms could be attributed to poor ventricular rate control and possible exacerbation of pulmonary obstruction. Hence, she would be first assigned to optimization of rate control, treatment of pulmonary obstruction, and lifestyle and risk factor modifications including weight reduction, all of which could have been initiated in the local health care center. ${ }^{48}$ Should she remain highly symptomatic after optimization of her medical condition and weight control, an advanced substrate evaluation using additional imaging could be undertaken to reconsider the choice between rate or rhythm control, but it is less likely that she would be scheduled for left atrial ablation.

\section{Limitations of the 4S-AF Characterization Scheme}

Identifying the most informative descriptor(s) for each of the domains relevant for the characterization of $\mathrm{AF}$ is challenging, and many diagnostic tools, risk stratification scores, ${ }^{49}$ and imaging techniques are currently being validated. Currently, the 4S-AF scheme mostly includes the AF-related descriptors that are easily obtainable in clinical practice, still acknowledging the possibility of more sophisticated assessment of all four domains.

The 4S-AF system does not provide information about bleeding risk, repeated cardioversions or AF ablations, prior and current antiarrhythmic drug therapy, etc. Nevertheless, the risk of bleeding is routinely assessed in patients considered for or taking OAC and should be noted in the medical records along with other features mentioned above. In addition, the 4S-AF currently does not reflect patientassessed symptoms or treatment burden, patient preferences, adherence to treatment, etc.

The 4S-AF structured characterization of AF pertains exclusively to clinically diagnosed $\mathrm{AF}$ and does not include so-called "subclinical AF" or atrial high rate episodes (AHREs) detected by implantable cardiac devices. However, when more evidence informing the management of AHRE with regards to stroke prevention become available, the $4 \mathrm{~S}-\mathrm{AF}$ scheme could be updated accordingly.

\section{Advantages of the 4S-AF Characterization Scheme}

As previously discussed, adherence to the $4 \mathrm{~S}-\mathrm{AF}$ scheme for characterization of AF patients would provide rapid, more concise, and clear communication among all physicians engaged in the management of a given AF patient, at all health care system levels, thus minimizing the risk of misunderstanding. Streamlining the assessment of AF-related features and AF patients via the use of the structured $4 \mathrm{~S}-\mathrm{AF}$ scheme would also facilitate treatment decision-making and could potentially reduce the costs of repeated expert consultations and/or unnecessary conducting various expensive 
diagnostic tests. In the future, the $4 \mathrm{~S}-\mathrm{AF}$ scheme could facilitate combining different data sets for research purposes.

\section{Concluding Remarks}

Importantly, the 4S-AF scheme has a great potential for future refinements as advances occur in the technology used for the assessment of AF-related features such as the burden of AF and the substrate for the arrhythmia. We, therefore, believe that the implementation of the $4 \mathrm{~S}-\mathrm{AF}$ scheme for AF patient characterization in routine clinical practice would substantially facilitate overall AF management and AF research.

Given the descriptors of AF included in the 4S-AF scheme, the characterization of AF patients using 4S-AF could also provide prognostic information, although the clinical utility and prognostic value of the $4 \mathrm{~S}-\mathrm{AF}$ scheme for the characterization of $\mathrm{AF}$ needs extensive validation in different $\mathrm{AF}$ cohorts and clinical settings.

\section{Conflict of Interest}

T.S.P. serves as a consultant for Bayer and Pfizer (no fees). G.Y.H.L. and G.B. received speaker fees of small amount from Medtronic, Boston, Biotronic, and Bayer.

\section{References}

1 Lewis T. Report Cxix. Auricular fibrillation: a common clinical condition. BMJ 1909;2(2552):1528

2 Lip G, Freedman B, De Caterina R, Potpara TS. Stroke prevention in atrial fibrillation: past, present and future. Comparing the guidelines and practical decision-making. Thromb Haemost 2017;117 (07):1230-1239

3 Piccini JP, Fauchier L. Rhythm control in atrial fibrillation. Lancet 2016;388(10046):829-840

4 Cosio FG, Aliot E, Botto GL, et al. Delayed rhythm control of atrial fibrillation may be a cause of failure to prevent recurrences: reasons for change to active antiarrhythmic treatment at the time of the first detected episode. Europace 2008;10(01):21-27

5 Kotecha D, Chua WWL, Fabritz L, et al; European Society of Cardiology (ESC) Atrial Fibrillation Guidelines Taskforce, the CATCH ME consortium and the European Heart Rhythm Association (EHRA). European Society of Cardiology smartphone and tablet applications for patients with atrial fibrillation and their health care providers. Europace 2018;20(02):225-233

6 Kirchhof P, Benussi S, Kotecha D, et al. 2016 ESC Guidelines for the management of atrial fibrillation developed in collaboration with EACTS. Europace 2016;18(11):1609-1678

7 January CT, Wann LS, Calkins H, et al. 2019 AHA/ACC/HRS focused update of the $2014 \mathrm{AHA} / \mathrm{ACC} / \mathrm{HRS}$ guideline for the management of patients with atrial fibrillation: a report of the American College of Cardiology/American Heart Association Task Force on Clinical Practice Guidelines and the Heart Rhythm Society in Collaboration With the Society of Thoracic Surgeons. Circulation 2019;140(02):e125-e151

8 Andrade JG, Verma A, Mitchell LB, et al; CCS Atrial Fibrillation Guidelines Committee. 2018 Focused Update of the Canadian Cardiovascular Society Guidelines for the Management of Atrial Fibrillation. Can J Cardiol 2018;34(11):1371-1392

9 Brieger D, Amerena J, Attia J, et al; NHFA CSANZ Atrial Fibrillation Guideline Working Group. National Heart Foundation of Australia and the Cardiac Society of Australia and New Zealand: Australian Clinical Guidelines for the Diagnosis and Management of Atrial Fibrillation 2018. Heart Lung Circ 2018;27(10):1209-1266
10 Gallagher MM, Camm J. Classification of atrial fibrillation. Am J Cardiol 1998;82(8A):18N-28N

11 Gallagher MM, Camm AJ. Classification of atrial fibrillation. Pacing Clin Electrophysiol 1997;20(06):1603-1605

12 Gallagher MM, Camm AJ. Long-term management of atrial fibrillation. Clin Cardiol 1997;20(04):381-390

13 Lévy S, Breithardt G, Campbell RW, et al; Working Group on Arrhythmias of the European Society of Cardiology. Atrial fibrillation: current knowledge and recommendations for management. Eur Heart J 1998;19(09):1294-1320

14 Lévy S, Novella P, Ricard P, Paganelli F. Paroxysmal atrial fibrillation: a need for classification. J Cardiovasc Electrophysiol 1995;6 (01):69-74

15 Camm AJ, Al-Khatib SM, Calkins H, et al. A proposal for new clinical concepts in the management of atrial fibrillation. Am Heart J 2012;164(03):292-302.e1

16 Hammond-Haley M, Providencia R, Lambiase PD. Temporal pattern/episode duration-based classification of atrial fibrillation as paroxysmal vs. persistent: is it time to develop a more integrated prognostic score to optimize management? Europace 2018;20 (FI_3):f288-f298

17 Wineinger NE, Barrett PM, Zhang Y, et al. Identification of paroxysmal atrial fibrillation subtypes in over 13,000 individuals. Heart Rhythm 2019;16(01):26-30

18 De With RR, Erküner Ö, Rienstra M, et al; RACE V Investigators. Temporal patterns and short-term progression of paroxysmal atrial fibrillation: data from RACE V. Europace 2020;22(08): 1162-1172

19 Lubitz SA, Benjamin EJ, Ruskin JN, Fuster V, Ellinor PT. Challenges in the classification of atrial fibrillation. Nat Rev Cardiol 2010;7 (08):451-460

20 Lip GYH. The ABC pathway: an integrated approach to improve AF management. Nat Rev Cardiol 2017;14(11):627-628

21 Chao TF, Lip GYH, Liu CJ, et al. Relationship of aging and incident comorbidities to stroke risk in patients with atrial fibrillation. J Am Coll Cardiol 2018;71(02):122-132

22 Chao TF, Lip GYH, Lin YJ, et al. Incident risk factors and major bleeding in patients with atrial fibrillation treated with oral anticoagulants: a comparison of baseline, follow-up and delta HAS-BLED scores with an approach focused on modifiable bleeding risk factors. Thromb Haemost 2018;118(04):768-777

23 Guo Y, Chen Y, Lane DA, Liu L, Wang Y, Lip GYH. Mobile health technology for atrial fibrillation management integrating decision support, education, and patient involvement: mAF app trial. Am J Med 2017;130(12):1388-1396

24 Diederichsen SZ, Haugan KJ, Brandes A, et al. Natural history of subclinical atrial fibrillation detected by implanted loop recorders. J Am Coll Cardiol 2019;74(22):2771-2781

25 Seligman WH, Das-Gupta Z, Jobi-Odeneye AO, et al. Development of an international standard set of outcome measures for patients with atrial fibrillation: a report of the International Consortium for Health Outcomes Measurement (ICHOM) atrial fibrillation working group. Eur Heart J 2020;41(10):1132-1140

26 Hijazi Z, Lindbäck J, Alexander JH, et al; ARISTOTLE and STABILITY Investigators. The $\mathrm{ABC}$ (age, biomarkers, clinical history) stroke risk score: a biomarker-based risk score for predicting stroke in atrial fibrillation. Eur Heart J 2016;37(20):1582-1590

27 Berg DD, Ruff CT, Jarolim P, et al. Performance of the ABC scores for assessing the risk of stroke or systemic embolism and bleeding in patients with atrial fibrillation in ENGAGE AF-TIMI 48. Circulation 2019;139(06):760-771

28 Ganesan AN, Chew DP, Hartshorne T, et al. The impact of atrial fibrillation type on the risk of thromboembolism, mortality, and bleeding: a systematic review and meta-analysis. Eur Heart J 2016;37(20):1591-1602

29 Al-Khatib SM, Thomas L, Wallentin L, et al. Outcomes of apixaban vs. warfarin by type and duration of atrial fibrillation: results from the ARISTOTLE trial. Eur Heart J 2013;34(31):2464-2471 
30 Hart RG, Pearce LA, Rothbart RM, McAnulty JH, Asinger RW, Halperin JLStroke Prevention in Atrial Fibrillation Investigators. Stroke with intermittent atrial fibrillation: incidence and predictors during aspirin therapy. J Am Coll Cardiol 2000;35(01):183-187

31 Nieuwlaat R, Dinh T, Olsson SB, et al; Euro Heart Survey Investigators. Should we abandon the common practice of withholding oral anticoagulation in paroxysmal atrial fibrillation? Eur Heart J 2008;29(07):915-922

32 Takabayashi K, Hamatani Y, Yamashita Y, et al. Incidence of stroke or systemic embolism in paroxysmal versus sustained atrial fibrillation: the Fushimi Atrial Fibrillation Registry. Stroke 2015;46(12):3354-3361

33 Mahajan R, Perera T, Elliott AD, et al. Subclinical device-detected atrial fibrillation and stroke risk: a systematic review and metaanalysis. Eur Heart J 2018;39(16):1407-1415

34 Wang SV, Rogers JR, Jin Y, et al. Stepped-wedge randomised trial to evaluate population health intervention designed to increase appropriate anticoagulation in patients with atrial fibrillation. BMJ Qual Saf 2019;28(10):835-842

35 Pastori D, Pignatelli P, Menichelli D, Violi F, Lip GYH. Integrated care management of patients with atrial fibrillation and risk of cardiovascular events: the $\mathrm{ABC}$ (Atrial fibrillation Better Care) Pathway in the ATHERO-AF Study Cohort. Mayo Clin Proc 2019; 94:1261-1267

36 Björkenheim A, Brandes A, Magnuson A, et al. Assessment of atrial fibrillation-specific symptoms before and 2 years after atrial fibrillation ablation: do patients and physicians differ in their perception of symptom relief? JACC Clin Electrophysiol 2017;3 (10):1168-1176

37 Potpara TS, Mihajlovic M, Zec N, et al. Self-reported treatment burden in patients with atrial fibrillation: Quantification, major determinants and implications for integrated holistic management of the arrhythmia. Europace 2020 (accepted)

38 Blomström-Lundqvist C, Gizurarson S, Schwieler J, et al. Effect of catheter ablation vs antiarrhythmic medication on quality of life in patients with atrial fibrillation: the CAPTAF randomized clinical trial. JAMA 2019;321(11):1059-1068

39 Staerk L, Sherer JA, Ko D, Benjamin EJ, Helm RH. Atrial fibrillation: epidemiology, pathophysiology, and clinical outcomes. Circ Res 2017;120(09):1501-1517

40 Staerk L, Wang B, Preis SR, et al. Lifetime risk of atrial fibrillation according to optimal, borderline, or elevated levels of risk factors: cohort study based on longitudinal data from the Framingham Heart Study. BMJ 2018;361:k1453

41 Brandes A, Smit MD, Nguyen BO, Rienstra M, Van Gelder IC. Risk factor management in atrial fibrillation. Arrhythm Electrophysiol Rev 2018;7(02):118-127

42 Donal E, Lip GY, Galderisi M, et al. EACVI/EHRA Expert Consensus Document on the role of multi-modality imaging for the evaluation of patients with atrial fibrillation. Eur Heart J Cardiovasc Imaging 2016;17(04):355-383

43 Hoit BD. Left atrial size and function: role in prognosis. J Am Coll Cardiol 2014;63(06):493-505

44 Khan MA, Yang EY, Zhan Y, et al. Association of left atrial volume index and all-cause mortality in patients referred for routine cardiovascular magnetic resonance: a multicenter study. J Cardiovasc Magn Reson 2019;21(01):4

45 Calkins H, Hindricks G, Cappato R, et al. 2017 HRS/EHRA/ECAS/ APHRS/SOLAECE expert consensus statement on catheter and surgical ablation of atrial fibrillation: executive summary. Europace 2018;20(01):157-208
46 Shade JK, Ali RL, Basile D, et al. Preprocedure application of machine learning and mechanistic simulations predicts likelihood of paroxysmal atrial fibrillation recurrence following pulmonary vein isolation. Circ Arrhythm Electrophysiol 2020;13 (07):e008213

47 Goette A, Kalman JM, Aguinaga L, et al; Document Reviewers. EHRA/HRS/APHRS/SOLAECE expert consensus on atrial cardiomyopathies: definition, characterization, and clinical implication. Europace 2016;18(10):1455-1490

48 Wijtvliet EPJP, Tieleman RG, van Gelder IC, et al; RACE 4 Investigators. Nurse-led vs. usual-care for atrial fibrillation. Eur Heart J 2020;41(05):634-641

49 Deng H, Bai Y, Shantsila A, Fauchier L, Potpara TS, Lip GYH. Clinical scores for outcomes of rhythm control or arrhythmia progression in patients with atrial fibrillation: a systematic review. Clin Res Cardiol 2017;106(10):813-823

50 Cosedis Nielsen J, Johannessen A, Raatikainen P, et al. Radiofrequency ablation as initial therapy in paroxysmal atrial fibrillation. N Engl J Med 2012;367(17):1587-1595

51 Morillo CA, Verma A, Connolly SJ, et al; RAAFT-2 Investigators. Radiofrequency ablation vs antiarrhythmic drugs as first-line treatment of paroxysmal atrial fibrillation (RAAFT-2): a randomized trial. JAMA 2014;311(07):692-700

52 Wazni OM, Marrouche NF, Martin DO, et al. Radiofrequency ablation vs antiarrhythmic drugs as first-line treatment of symptomatic atrial fibrillation: a randomized trial. JAMA 2005;293 (21):2634-2640

53 Pappone C, Augello G, Sala S, et al. A randomized trial of circumferential pulmonary vein ablation versus antiarrhythmic drug therapy in paroxysmal atrial fibrillation: the APAF Study.J Am Coll Cardiol 2006;48(11):2340-2347

54 Jaïs P, Cauchemez B, Macle L, et al. Catheter ablation versus antiarrhythmic drugs for atrial fibrillation: the A4 study. Circulation 2008; 118(24):2498-2505

55 Wilber DJ, Pappone C, Neuzil P, et al; ThermoCool AF Trial Investigators. Comparison of antiarrhythmic drug therapy and radiofrequency catheter ablation in patients with paroxysmal atrial fibrillation: a randomized controlled trial. JAMA 2010;303 (04):333-340

56 Stabile G, Bertaglia E, Senatore G, et al. Catheter ablation treatment in patients with drug-refractory atrial fibrillation: a prospective, multi-centre, randomized, controlled study (Catheter Ablation For The Cure Of Atrial Fibrillation Study). Eur Heart J 2006;27(02):216-221

57 Oral H, Pappone C, Chugh A, et al. Circumferential pulmonaryvein ablation for chronic atrial fibrillation. N Engl J Med 2006;354 (09):934-941

58 Packer DL, Kowal RC, Wheelan KR, et al; STOP AF Cryoablation Investigators. Cryoballoon ablation of pulmonary veins for paroxysmal atrial fibrillation: first results of the North American Arctic Front (STOP AF) pivotal trial. J Am Coll Cardiol 2013;61(16): 1713-1723

59 Forleo GB, Mantica M, De Luca L, et al. Catheter ablation of atrial fibrillation in patients with diabetes mellitus type 2: results from a randomized study comparing pulmonary vein isolation versus antiarrhythmic drug therapy. J Cardiovasc Electrophysiol 2009;20 (01):22-28

60 Mont L, Bisbal F, Hernández-Madrid A, et al; SARA investigators. Catheter ablation vs. antiarrhythmic drug treatment of persistent atrial fibrillation: a multicentre, randomized, controlled trial (SARA study). Eur Heart J 2014;35(08):501-507 